\title{
In Situ Treatment of Thermal RF Plasma Processed Nanopowders to Control their Agglomeration and Dispersability
}

\author{
M. Leparoux $\cdot$ Y. Leconte $\cdot$ A. Wirth $\cdot$ Th. Buehler
}

Received: 13 August 2010/Accepted: 1 October 2010/Published online: 20 October 2010

(C) Springer Science+Business Media, LLC 2010

\begin{abstract}
Titanium carbonitride nanoparticles have been produced in an inductively coupled thermal plasma and subsequently modified using a surfactant that has been deposited in situ on their surface in-flight. The surfactant was injected in the reactor while the nanoparticles are still dispersed in the gas phase, allowing the coating of primary particles instead of the corresponding agglomerates. In contrast to naked TiCN nanoparticles, the surfactant coated particles could be readily dispersed in water with a short ultrasonic treatment and built up no large agglomerates as proved by Photon Correlation Spectroscopy measurements. The investigated surfactants seem, however, to undergo a chemical modification and/or a thermal degradation at the surface of the TiCN nanoparticles.
\end{abstract}

Keywords Nanoparticles - Surface modification - Thermal plasma - Dispersion - Safety

\section{Introduction}

The potential of nanoparticles has been widely highlighted due to their generally different properties when compared to the bulk material $[1,2]$. If these specific properties are mainly induced by a high surface to volume ratio, this large specific surface area also promotes their agglomeration and sometimes their irreversible aggregation. In gas phase synthesis processes, the particles are generally separated from the gas phase on filter surfaces where they agglomerate in a particle-cake. Moreover in the gas phase, the large Brownian motion

M. Leparoux ( $\bowtie)$

Advanced Materials Processing, Empa, Swiss Federal Laboratories for Materials Science and Technology, Feuerwerkerstrasse 39, 3602 Thun, Switzerland

e-mail: marc.leparoux@empa.ch

Y. Leconte

CEA, IRAMIS, SPAM, LFP, 91191 Gif/Yvette, France

A. Wirth - Th. Buehler

School for Life Sciences, University of Applied Sciences,

Gruendenstrasse 40, 4132 Muttenz, Switzerland 
of the produced nanoparticles increases the collision frequency and thus the probability for two or more nanoparticles to stick and grow together. In the case of weak bridging between the particles, such as van der Waals forces, the agglomeration is called soft agglomeration whereas in the case of a more stable bridge formation, like sintering, the agglomerates are called hard agglomerates or aggregates. In this latter, the nanoparticles generally lose their inherent and unique properties and behave in similar way as micro-sized particles [3, 4]. For composite materials reinforced with nanoparticulate materials, the achievement of strengthening can be only obtained when the nanoparticles are homogeneously well dispersed in the matrix. Moreover, when it comes to sintering in order to prepare nanostructured ceramics, the distribution, the size and the porosity of the nanoparticles in the green body appears to be a key issue to obtain high densification while avoiding excessive grain growth. Therefore, the agglomeration state of the nanopowders should be controlled in addition to their size and particle size distribution.

To promote a good dispersion of nanoparticles, beside the control of the synthesis process itself, surfactants are usually mixed to the agglomerated particles ex-situ in a solution under mechanical or ultrasonic treatment [5-9].

The large molecules of the surfactant adsorb on the surface of single particles preventing the direct contact between the particles and the post generation of agglomerates [6-8]. Moreover, depending on the chain length of the surfactant, inter-particle interaction can be avoided through steric hindrance [5]. If, however, the particles are not dispersed at the position where the surfactant solution is introduced in the reactor, the polymer will adsorb mostly on the external surface of the formed agglomerates making a further dispersion difficult.

Qin and Coulombe [10] reported a dual-plasma process consisting of two sub-steps in a single reactor: the copper nanoparticles synthesis through arc evaporation and vapour condensation followed by the in-flight deposition of an organic layer onto the nanoparticles through plasma polymerisation. A 3-10 nm thick polymer-like film was deposited from the plasma polymerization of the monomer gas $\mathrm{C}_{2} \mathrm{H}_{6}$. However, no dispersion behaviour is reported for the coated nanoparticles.

Hakim et al. [11] investigated the modification of interparticle forces for nanoparticles using atomic layer deposition (ALD). They showed that whatever the investigated conditions, the $\mathrm{TiO}_{2}$ and $\mathrm{SiO}_{2}$ nanoparticles coated with an ultra thin alumina film had a higher agglomeration state and consequently a lower flowability than the non-treated nanoparticles.

This paper is reporting the ability of an inductively coupled thermal plasma (ICP) to produce titanium carbonitride nanoparticles with a soft agglomeration behaviour and the subsequent in situ in-flight deposition of a surfactant on primary nanoparticles promoting their dispersability. The aim is to integrate several processing steps (here nanoparticle synthesis and in-flight coating) to reduce the number of ex-situ treatments before the particles can be used for an application. This has also positive consequences considering safety issues induced by any manipulation of nanopowders.

\section{Experimental}

\section{Plasma Synthesis of the TiCN Nanoparticles}

The nanoparticles were produced by evaporating a solid precursor in a thermal plasma followed by rapid condensation of the gas phase [12-15]. The precursors are generally 
microscale particles but liquids and also gases may be used. In this study, TiCN powders (Ti(C,N) 50/50 grade B, H.C. Starck, Germany) with a Fisher sub-sieve size (FSSS) of $1.4 \mu \mathrm{m}$ have been used as starting material. The thermal plasma is an ICP operating at pressures typically between 20 and $80 \mathrm{kPa}$ with the RF generator working at $13.56 \mathrm{MHz}$. The nanoparticles synthesis was performed according to the process reported by Leparoux et al. [14] and described in details elsewhere. The reactor is shown in Fig. 1 and the parameters used for the synthesis of TiCN nanoparticles are summarized in Table 1 . The precursor was introduced into the plasma torch using a dense phase convey powder feeder system (DACS, Switzerland) at a feed rate of $0.7 \mathrm{~g} \mathrm{~min}^{-1}$. The conditions for the quenching, i.e. the rapid cooling of the particles in the gas phase preventing their further growth, were adapted to the investigated TiCN material. Thus the quenching was performed at a distance $z=110 \mathrm{~mm}$ from the torch exhaust with 42 slpm of argon [14].

Using an in-line sampling system, nanoparticles have been collected in situ on a TEM grid during their plasma synthesis. The system is flanged onto the different view-ports of the reactor chamber ( $b$ in Fig. 1 links). Therewith using a high resolution electron microscope, the nanoparticle growth and agglomeration state can be followed as a function of the process parameters. Figure 2 shows the scanning electron micrographs illustrating the agglomeration of ICP processed silicon nanoparticles at two different positions from the torch exhaust in the reactor. In this case, the nanoparticles were synthesised in the same reactor under similar conditions as for the present study starting from $30 \mu \mathrm{m}$ silicon precursor particles.

These SEM pictures show that in the upper part of the plasma chamber, the nanoparticles are dispersed in the gas phase-isolated primary particles are observed on the TEM grid (Fig. 2a), whereas close to the filter on which the particle are normally collected, the nanoparticles form mainly agglomerates (Fig. 2b). By sampling particles at different heights in the reactor chamber, it has been observed that the particles are normally
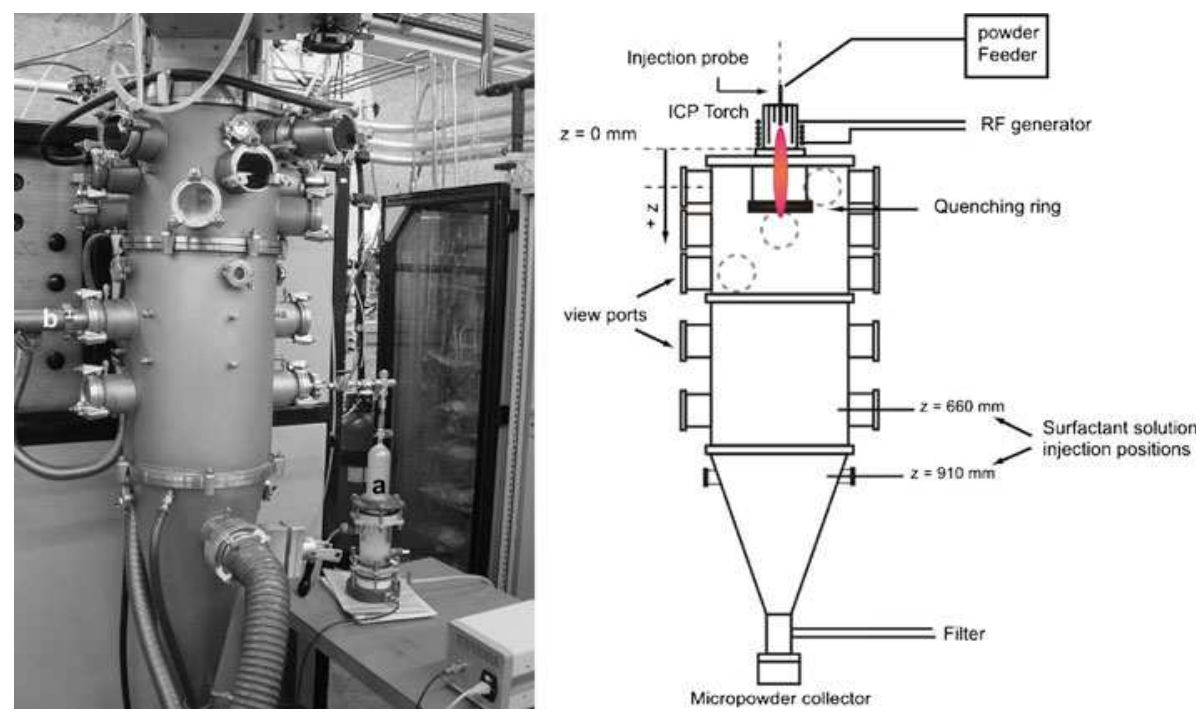

Fig. 1 Picture and scheme of the plasma reactor used for the synthesis of nanoparticles. The nebuliser for the injection of the surfactant is shown on the right (a) in the picture above whereas the in-line sampling system is seen on the left (b) 
Table 1 Process parameters used for the synthesis of TiCN nanoparticles

\begin{tabular}{ll}
\hline Carrier gas $(\mathrm{slpm})=5 \mathrm{Ar}$ & PL-35 torch (Tekna Plasma Systems, Ca) \\
Central gas $(\mathrm{slpm})=12 \mathrm{Ar}$ & Input RF-power $=15.5 \mathrm{~kW}$ \\
Sheath gas $(\mathrm{slpm})=60 \mathrm{Ar}+6 \mathrm{~N}_{2}$ & Pressure $=40 \mathrm{kPa}$ \\
Injector position $62 \mathrm{~mm}$ above the torch exhaust & Precursor powder under Ar \\
\hline
\end{tabular}
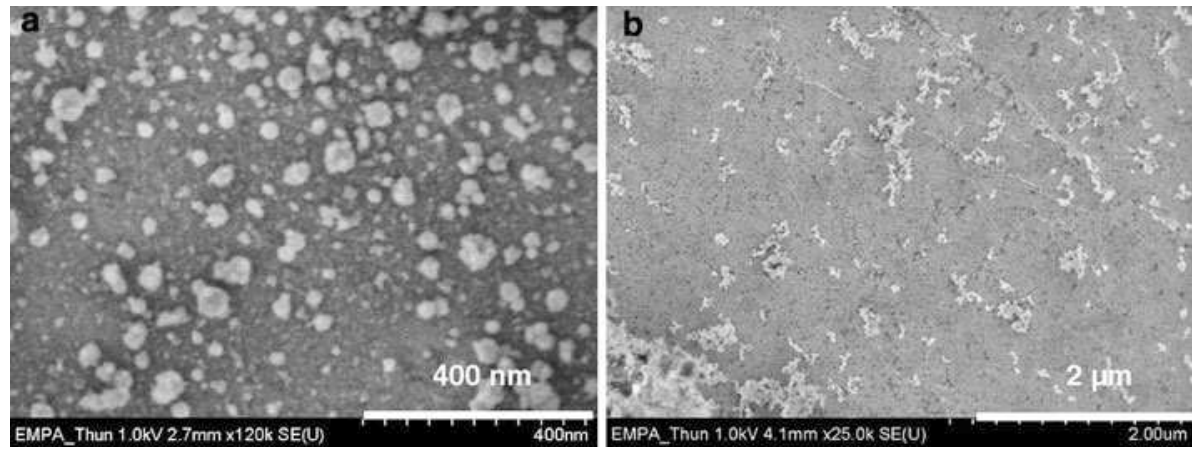

Fig. 2 Si-nanoparticles collected in-line on a TEM-grid in the reactor during processing. a At $z=260 \mathrm{~mm}$ from the torch exhaust the primary particles with an average size around 30-40 nm seem non-agglomerated whereas, $\mathbf{b}$ at $z=3,500 \mathrm{~mm}$ close to the filtration unit, the primary particles form mainly agglomerates

dispersed in the gas phase on a distance of about $1 \mathrm{~m}$ from the torch exhaust. This distance corresponds roughly to the top of the cone-shape modular chamber in the used reactor (see Fig. 1). The restriction of the chamber increases the particle density, promotes the in-flight collisions between nanoparticles and thus their agglomeration.

To ensure an effective dispersion of the primary particles, it is then important to coat the nanoparticles before the agglomeration process starts in the gas phase. The surfactant should then be introduced in the upper part of the ICP chamber before the cone-shape area.

\section{In Situ In-Flight Coating of the Nanoparticles}

Using the same process parameters as described in the previous section, the in-flight deposition of a surfactant on the surface of the nanoparticles directly after their synthesis has been studied. Therefore, three different aqueous surfactant solutions (Table 2) were diluted in water or ethanol to achieve a concentration of $10 \mathrm{vol} \%$. This diluted solution was then placed in a nebuliser equipped with a piezoelectric pellet generating fine droplets above the liquid surface. Optical diffraction measurements performed on toluene droplets produced by the nebulizer used in this study, revealed a mean droplet size around $8 \mu \mathrm{m}$. Argon was used as carrier gas for transporting these droplets into the reactor through a single injector with an inner diameter of $4 \mathrm{~mm}$. The distance between the nebuliser and the reactor was kept as short as possible to limit the deposition of the droplets in the feeding line and the injector. Two injection positions were investigated at $z=660$ and $910 \mathrm{~mm}$ in height from the torch exhaust (Fig. 1). The lowest injection point at $910 \mathrm{~mm}$ corresponds to the maximal distance available before agglomeration takes place in the reactor, while the highest point was selected in order to avoid high temperatures that could completely destroy the organic surfactants (see the temperature profiles measured with a thermocouple 
Table 2 Surfactants investigated

\begin{tabular}{lll}
\hline Name & Supplier & Information given by the supplier \\
\hline RHEOSPERSE 1302 & Coatex (France) & 35 wt $\%$ of branched acrylic copolymer in water \\
RHEOSPERSE 1420 & Coatex (France) & $26 \mathrm{wt} \%$ of branched acrylic copolymer in water \\
RHEOSPERSE 205 & Coatex (France) & $40 \mathrm{wt} \%$ of linear acrylic copolymer in water \\
\hline
\end{tabular}

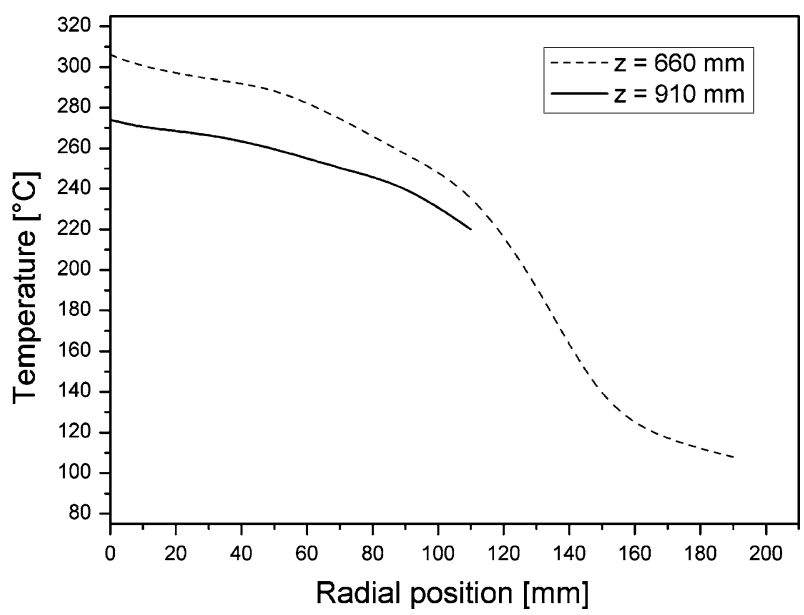

Fig. 3 Temperature profiles for the two surfactant injection points measured in the reactor from the chamber axis under plasma processing but powder-free conditions

at these positions under processing but powder-free conditions in Fig. 3). The radial distances of the injection points from the axis of the reactor were 190 and $110 \mathrm{~mm}$ for $z=660$ and $910 \mathrm{~mm}$ positions respectively. The difference of the radial positions is due to the conic shape of the bottom of the reactor starting already above $z=910 \mathrm{~mm}$. The transported liquid quantity was estimated by weighting the remaining solution after completion of the experiment. The investigated parameters are summarized in Table 3.

Photon correlation spectroscopy (PCS, Malvern HS1000) also called Dynamic Light Scattering (DLS) has been used to measure the particles size distribution and subsequently the influence of the surfactant type on the nanoparticles dispersability. Therefore, the nanopowders were dispersed in distilled water and then immersed in a conventional ultrasonic (US) bath for $5 \mathrm{~min}$. After a first PCS measurement, a high energy $(700 \mathrm{~W}$, $15 \mathrm{ml}$ ) US probe was then introduced into the dispersion and PCS analyses were performed after sonication during 2 and $30 \mathrm{~min}$. As PCS results are annotated with modes of the particle size distribution (using variable widths determined by the software), in the following, the indicated mean particle size is the average value of the main mode. A high resolution field emission scanning electron microscope (Zeiss, SUPRA ${ }^{\mathrm{TM}_{4}} 4$ ) was used for the characterization of the produced particles and has supported the observation on the particle size distribution and the agglomeration feature. Thermal analyses (Netzsch STA 409) were also performed in order to observe the weight changes with the temperature of the produced powders under a dynamic argon flow. Finally infrared (IR) spectroscopy (Digilab Varian FTS 700) was performed aiming at the characterization of the chemical bonding changes at the surface of the particles after aerosol treatment. 
Table 3 In-flight coating parameters

\begin{tabular}{llllllll}
\hline $\mathrm{N}^{\circ}$ & Surfactant & $\begin{array}{l}\text { Dilution } \\
\text { medium }\end{array}$ & $\begin{array}{l}\text { Injection } \\
\text { position } \\
(z \mathrm{~mm})\end{array}$ & $\begin{array}{l}\text { Carrier gas } \\
\text { nebuliser } \\
\left(\mathrm{N}_{2} \mathrm{slpm}\right)\end{array}$ & $\begin{array}{l}\text { Solution } \\
\text { feed rate } \\
\left(\mathrm{g} \mathrm{min}^{-1}\right)\end{array}$ & $\begin{array}{l}\text { Powder } \\
\text { feed rate } \\
\left(\mathrm{g} \mathrm{min}^{-1}\right)\end{array}$ & $\begin{array}{l}\text { Powder/ } \\
\text { solution }\end{array}$ \\
\hline 1 & RHEOSPERSE 1302 & Water & 910 & 6 & 0.40 & 0.7 & 1.8 \\
2 & RHEOSPERSE 1302 & Water & 910 & 2.5 & 0.15 & 0.75 & 5.0 \\
3 & RHEOSPERSE 1302 & Ethanol & 910 & 6 & 0.6 & 0.6 & 1.0 \\
4 & RHEOSPERSE 1302 & Ethanol & 660 & 6 & 0.7 & 1.7 & 2.4 \\
5 & RHEOSPERSE 1420 & Water & 660 & 6 & 0.2 & 1.0 & 5.0 \\
6 & RHEOSPERSE 1420 & Water & 660 & 10 & 0.15 & 0.8 & 5.7 \\
7 & RHEOSPERSE 205 & Water & 660 & 6 & 0.4 & 1.1 & 2.8 \\
8 & RHEOSPERSE 205 & Water & 910 & 6 & 0.2 & 0.7 & 3.5 \\
\hline
\end{tabular}

\section{Results}

Raw TiCN Nanoparticles

PCS and SEM analyses performed on filter collected powders show that the produced nanopowders have an average primary particle size around 20-25 $\mathrm{nm}$ with a narrow size distribution from about 15 to $45 \mathrm{~nm}$ (Figs. 4, 5). However the collected particles are agglomerated after the plasma processing; the PCS measurements (Fig. 4) reveal an average particle size around $300 \mathrm{~nm}$ with a small contribution centred around 1.6-1.8 $\mu \mathrm{m}$ after dispersion in water and low energy US treatment (bath) for $5 \mathrm{~min}$. After $2 \mathrm{~min}$ under powerful US probe treatment, the mean grain size decreases to $200 \mathrm{~nm}$ and the micro-sized contribution disappears. Nevertheless, 30 min duration of this US probe dissociation was found necessary in order to reach a mean grain size in good agreement with mean primary grain size determined by SEM. It seems that the raw nanopowders have two kinds of agglomerates; the soft ones are already dispersed after only 2 min probe treatment while some stronger ones require longer sonication.

The PCS measured primary particle size around $20-30 \mathrm{~nm}$ are validated by SEM characterization (Fig. 5) and some large agglomerates are still observed. At high magnification, no sintering necks were observed between the nanoparticles meaning that the

Fig. 4 Photon correlation spectroscopy measurements of the produced TiCN nanopowders dispersed in water as a function of different ultrasonic treatments

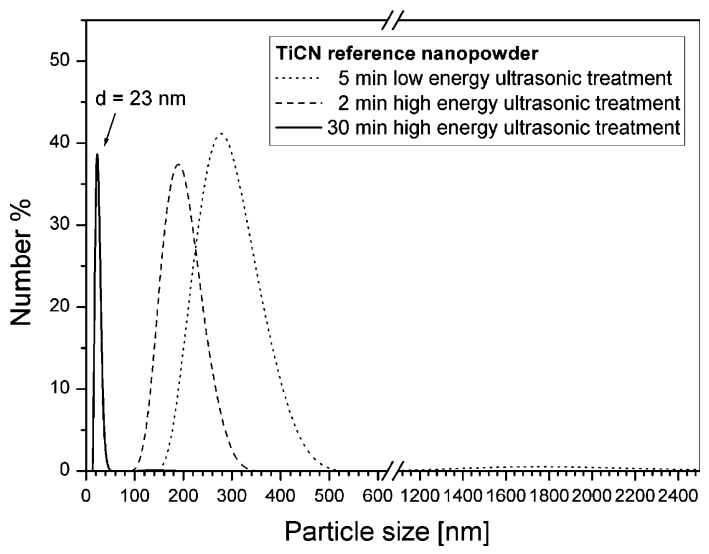



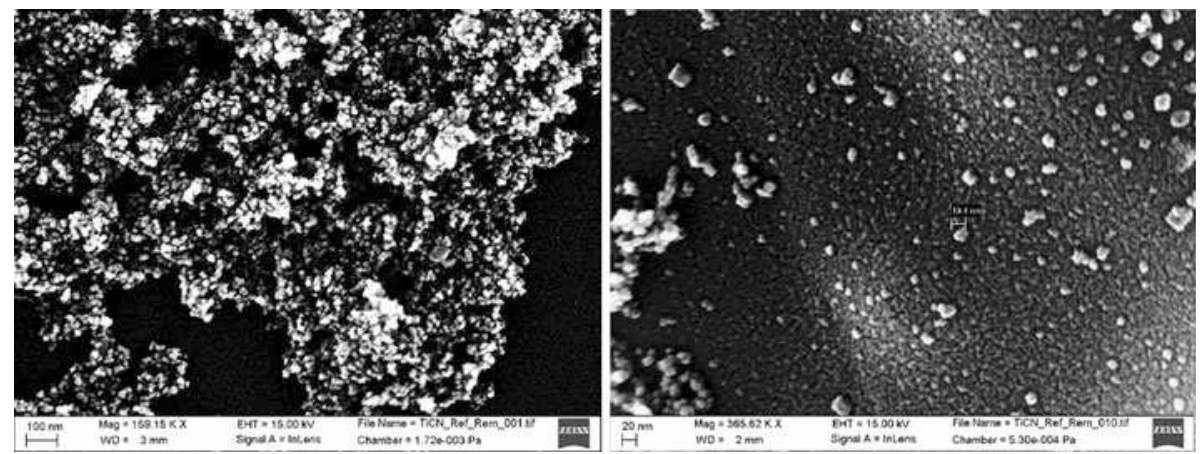

Fig. 5 SEM pictures of the ICP processed TiCN nanopowders without any surfactant

agglomerates may be only maintained by van der Waals forces (soft agglomerates). For these observations, the nanoparticles were dispersed in ethanol and a droplet of this dispersion was deposited on a freshly cleaved mica substrate. After evaporation of ethanol, the substrate was sputtered using a gold/palladium target and then introduced in the scanning electronic microscope.

\section{In Situ In-Flight Coated TiCN Nanopowders}

The deposition of a surfactant on the nanoparticles has been performed under the same plasma processing conditions described above, the surfactant being introduced below the processing zone at either $z=660$ or $910 \mathrm{~mm}$ from the torch exhaust (Table 3). At these injection positions far away from the processing zone (quenching takes place at $z=110 \mathrm{~mm}$ ), and considering the moderate flow rates used to transport the surfactant solution, the nanoparticles synthesis should not be influenced. The size and the size distribution of the primary particles are thus expected to be similar to the raw particles, with a mean size around 20-30 $\mathrm{nm}$ (Fig. 4).

From Table 3 it appears that an increase of the carrier gas flow through the nebuliser from 2.5 to $6 \mathrm{slpm}$ leads to an increase of the surfactant solution quantity introduced in the plasma reactor. However a further increase from 6 to $10 \mathrm{slpm}$ rather leads to slight decrease of the surfactant solution feed rate. This could be ascribed to the aerodynamic path of the droplets in the aerosol generator that changes with the carrier gas flow rate, promoting the deposition of droplets on the nebuliser and injector walls for high flow rates. The solution consumption is also limited by the aerosol production which is itself limited by the piezoelectric pellet power.

The dispersability has been assessed by PCS measurements of the particle size distribution as a function of the sonication route and duration of dispersions. The results are presented in Figs. 6 and 7.

First of all, even after short treatment in US bath $(5 \mathrm{~min})$, no micro-sized agglomerates could be detected in the surfactant treated samples as opposed to the bare powders that contain large agglomerates (around 1.5-2 $\mu \mathrm{m}$ ). Furthermore, the measured agglomerates size appears generally smaller than the equivalent one measured for raw nanopowders, showing the efficiency of the in situ treatment in avoiding the stabilization of big agglomerates. 


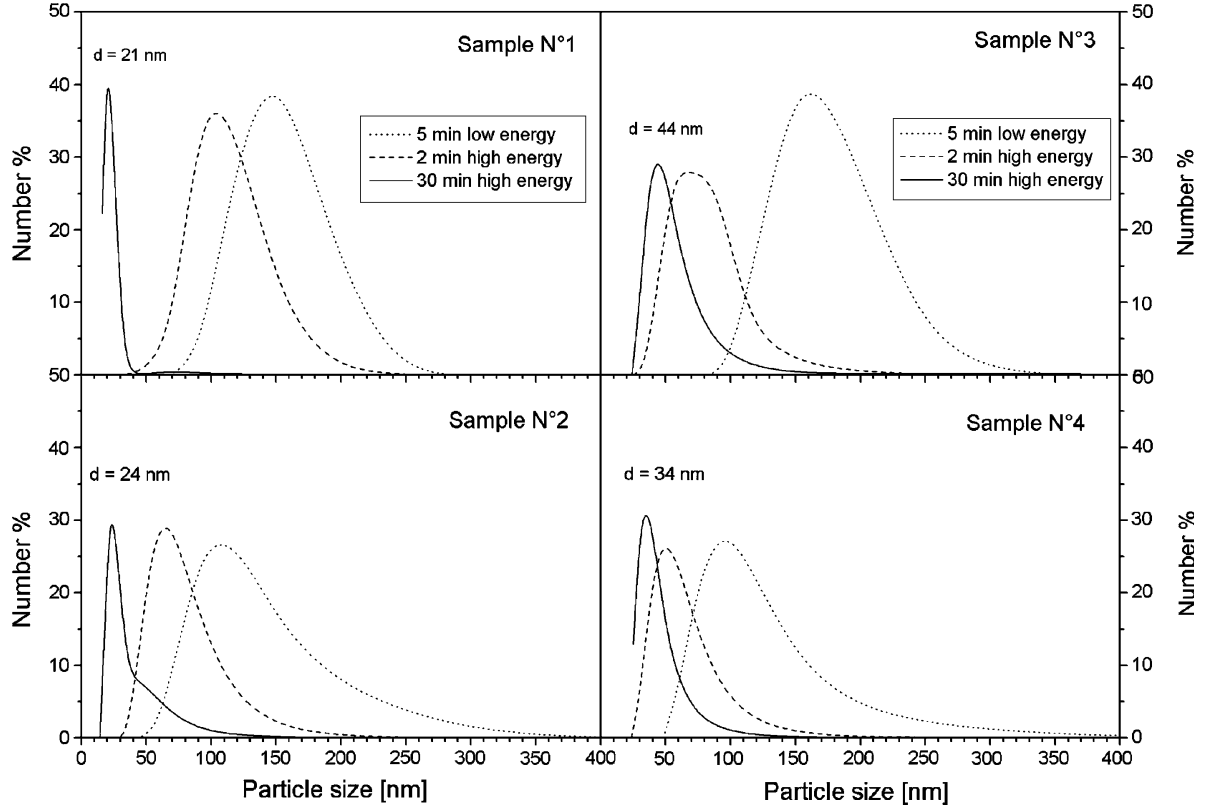

Fig. 6 Particle size distribution of samples 1-4 treated with a branched acrylic copolymer (RHEOSPERSE 1302) as a function of US treatments. The surfactant solution was further diluted to achieve a concentration of $10 \mathrm{vol} \%$ either in water (samples 1 and 2) or in ethanol (samples 3 and 4)

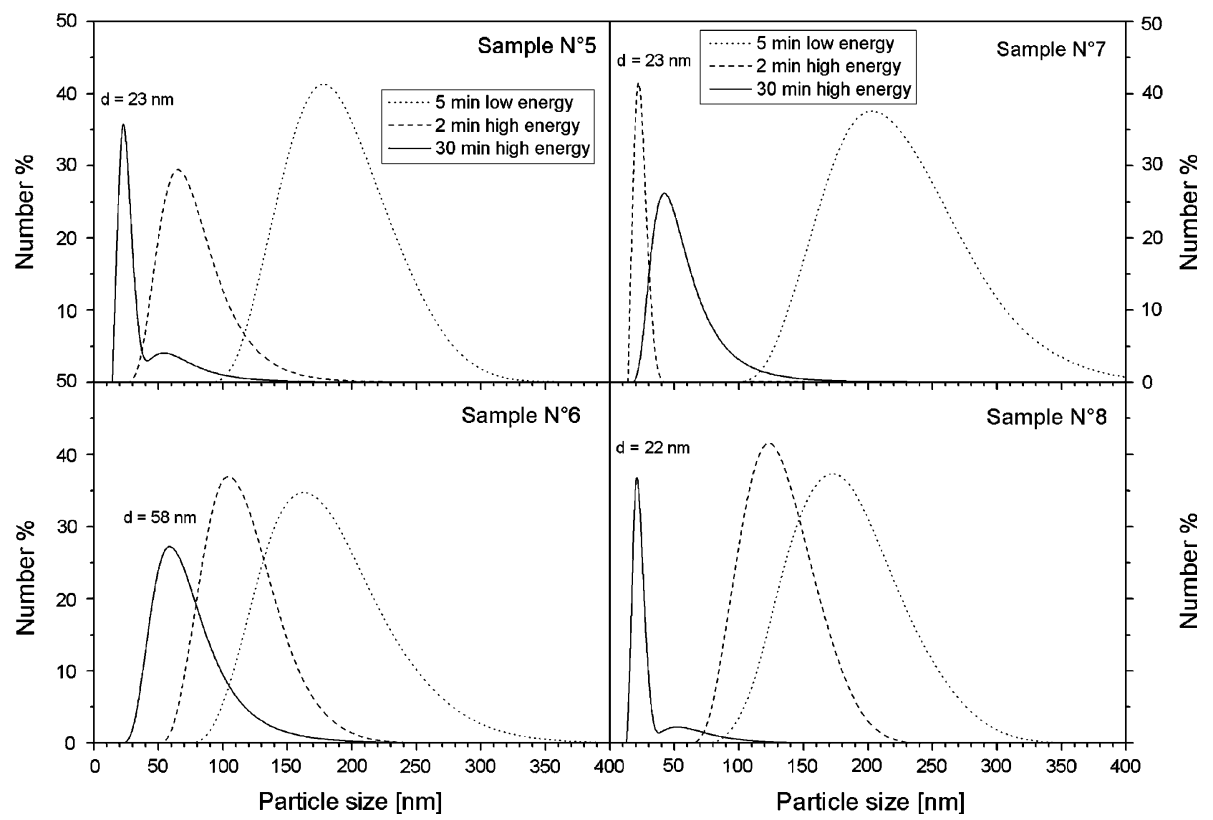

Fig. 7 Particle size distribution of samples 5-8 measured by PCS as a function of different ultrasonication treatments 
Sample 2 should contain less branched acrylic copolymer than sample 1, and its agglomerates mean size is slightly lower after $5 \mathrm{~min}$ in the US bath, and also after $2 \mathrm{~min}$ using US probe. However after $30 \mathrm{~min}$ US probe, for both samples the primary nanoparticles with a mean size around $20-25 \mathrm{~nm}$ are observed. Some agglomerates with a size around $50 \mathrm{~nm}$ are however remaining in sample 2 .

When the surfactant solution is diluted into ethanol (sample 3) instead of water (samples 1 and 2), and injected in the reactor at the same position, a good dispersion of the primary particles seems to be more difficult. Even after $30 \mathrm{~min}$ of sonication at high energy, the primary particles were not detected but agglomerates with a mean size around $40 \mathrm{~nm}$. Moreover, the agglomerate size distribution remains broader than for sample 1 functionalised from aqueous solutions with a comparable concentration. It is worth to note that the investigated surfactant solutions are recommended for aqueous suspensions. Therefore, the better dispersability of the nanoparticles in water than in ethanol is then not surprising. From all the samples investigated in this study, sample 3 should contain the highest surfactant relative concentration at the surface of the TiCN nanoparticles (mass ratio powder/surfactant is close to one).

For sample 4, compared to sample 3, the surfactant solution was injected in a smaller quantity relative to the powder feeding rate and also, at a higher position in the chamber $(z=660 \mathrm{~mm})$, meaning closer to the particle synthesis zone and at higher temperature. These conditions seem to be beneficial for an easy dispersability of the nanopowders as particles around $30 \mathrm{~nm}$ could then be detected and the observed agglomerates were generally smaller.

For sample 5, the initial branched acrylic copolymer concentration in the starting solution was 26 vol.\% instead of $35 \mathrm{vol} . \%$ for sample 2, but as the powder feed rate was lower in this latter sample the final powder/solution ratio is the same in both samples. This enables the comparison of samples 2 and 5 in order to study the effect of the injection position for the surfactant. After a soft sonication treatment in the bath for $5 \mathrm{~min}$, the agglomerates of sample 2 are smaller than the ones of sample 5 for which the surfactant solution was injected at a higher position. However, when dispersed with the high energy US probe, whatever the injection position, similar agglomeration behaviours were observed for these two samples.

Among all the samples, sample 6 is the one with the theoretical lowest surfactant concentration (lowest initial polymer concentration and highest powder mass/surfactant solution mass ratio). The mean particle size remains about $50-60 \mathrm{~nm}$ even after $30 \mathrm{~min}$ high energy ultrasonic treatment and the average agglomerates size after 2 min is also relatively high at about $100 \mathrm{~nm}$. It seems then that there is an optimum for the surfactant concentration; at too low or too high concentrations more ultrasonic energy is required to disperse the primary nanoparticles.

In samples 7 and 8, a linear acrylic polymer has been used as surfactant. The solution has been introduced at $660 \mathrm{~mm}$ from the torch exhaust for sample 7 and $910 \mathrm{~mm}$ for sample 8 . The relative concentrations (mass ratios between powder and surfactant solution) are in the same order, just slightly higher in sample 7 . For this later, primary nanoparticles are already observed after only 2 min sonication at high energy. Further US treatment seems to promote agglomeration of the particles again. When compared to previous samples covered by a branched acrylic copolymer, sample 7 demonstrates a better efficiency of the linear copolymer. Sample 8 shows a similar dispersability behaviour as the previous investigated samples and primary nanoparticles could be observed after $30 \mathrm{~min}$ sonication. While no significant influence of the injection position was observed for the 
branched polymer, it seems that a better dispersability efficiency is reached upon introducing the linear polymer at higher position in the reactor.

Among all the samples investigated in this study, sample 7 presents the best and the easiest dispersability. Therefore, this sample has been further characterized by scanning electron microscopy, thermal analysis and Fourier transform infrared (FTIR) spectroscopy to detect the presence of the surfactant at the surface of the in-flight treated particles.

For the SEM characterisations, the collected particles were here also first dispersed in ethanol and then deposited on a freshly cleaved mica substrate. Figure 8 shows that the primary particles in sample 7 have enough freedom motion to form by capillary forces a more spread layer on the mica substrate. During the evaporation of ethanol, the modified nanoparticles re-arrange themselves forming quasi-round agglomerates. This different behaviour compared to the raw TiCN nanoparticles shown in Fig. 5 can be explained by the presence of surfactant molecules at the surface of the nanoparticles. No sintering necks were observed in high resolution scanning microscopy.

Thermo-gravimetric analyses (TGA) have been performed up to $550^{\circ} \mathrm{C}$ under argon atmosphere. Figure 9 shows typical results obtained for sample 7 together with a comparison with raw TiCN nanopowders. The weight loss in the temperature range from 25 to $170^{\circ} \mathrm{C}$ is attributed mostly to the evaporation of moisture and residual water. The exothermic behaviour between $170^{\circ} \mathrm{C}$ and $370-450^{\circ} \mathrm{C}$ coupled with a significant weight loss is due to the decomposition and the carbonization of the organic surfactant. For temperatures above $450^{\circ} \mathrm{C}$, a moderate weight gain is observed and this is attributed to the oxidization of titanium due to the presence of some oxygen traces in the TGA chamber. Because of the high specific surface area of the nanopowders, titanium based nanomaterial is indeed much more sensitive, compared to its bulk form, to any oxygen present in the surrounding atmosphere. None of the samples showed a protection against oxidation at these high temperatures; the weight gain increase was comparable for all the investigated nanoparticles. The polymer film is damaged at such temperatures and cannot prevent a further oxidation.

Even if the TGA show that an organic substance is present at the surface of the nanoparticles, a clear comparison between the different samples is however difficult. This is due to the moisture concentration at the surface of the nanoparticles that is certainly dependent on the nature, the concentration and the integrity of the surfactant. Additionally, as mentioned previously the flow feed rate of the surfactant solution was only poorly
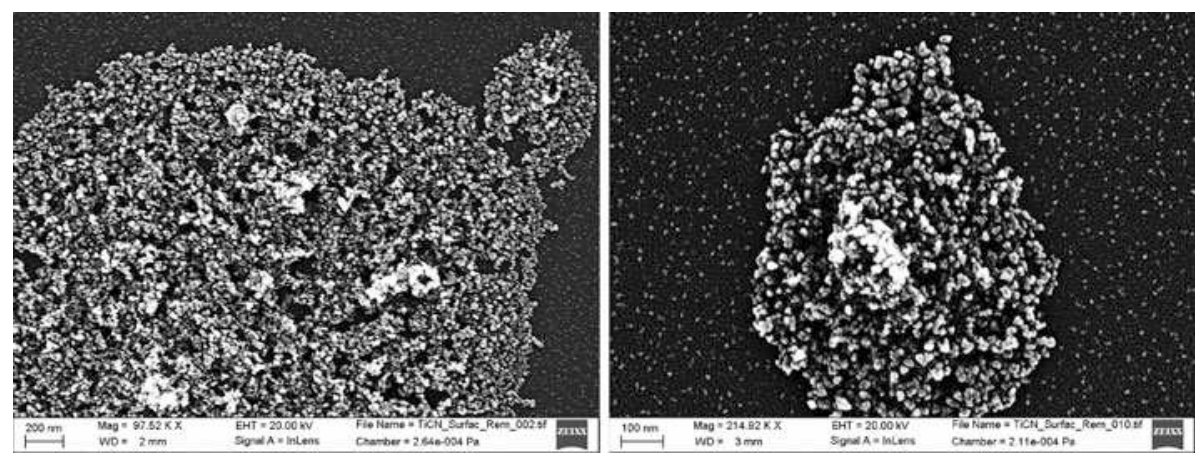

Fig. 8 SEM observation of the in-flight coated TiCN particles (sample 7) 
Fig. 9 Thermal gravimetric analyses of the ICP processed TiCN nanopowders with and without any surfactant

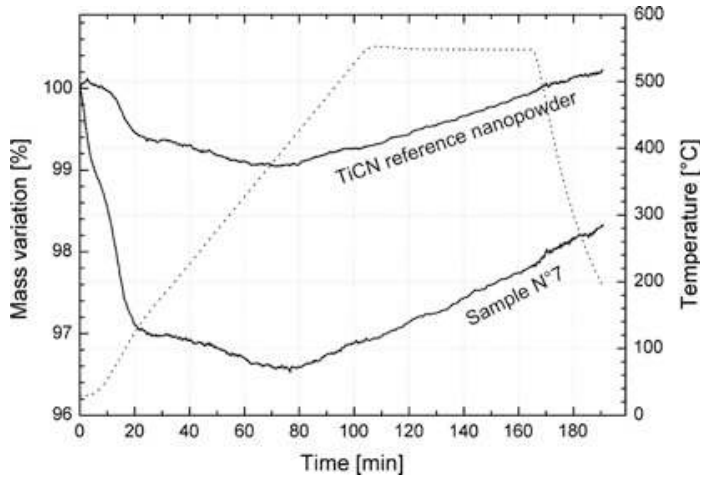

controlled due to some condensation in the feeding line. As the thermal degradation of the organic molecule by the chamber heat is not known for each sample, no clear relationship can be established between the weight loss in TGA and the surfactant content of the powders.

Figure 10 shows comparative FTIR spectra of sample 7 and non-coated TiCN nanoparticles. The IR signature of the surfactant solution has been also drawn for comparison.

The absorption bands observed in the range $2,000-2,400 \mathrm{~cm}^{-1}$ are attributed to $\mathrm{C} \equiv \mathrm{N}$ bands. The surfactant has a strong absorption band around 1,550 and 1,400-1,450 $\mathrm{cm}^{-1}$ as well as a broad band around $3,000-3,400 \mathrm{~cm}^{-1}$. The IR spectrum of surfactant coated TiCN nanoparticles in sample 7 shows, beside the standard TiCN bands, additional ones in the 3,200-3,300 and 1,300-1,600 $\mathrm{cm}^{-1}$ regions. These bands can be identified in the IR spectrum of REOSPERSE 205 with different shapes. This seems to indicate that the surfactant underwent a modification at the surface of the hot nanoparticles or a thermal degradation in the gas phase during the injection in the reaction chamber. The temperature at the injection point increased indeed from about $200^{\circ} \mathrm{C}$ at the injector outlet to more than $300^{\circ} \mathrm{C}$ in the middle of the chamber (Fig. 3). Nevertheless, the short residence time of the species in this region (a few ms) avoids a complete decomposition of the surfactant molecules at such high temperatures.

Fig. 10 FTIR spectra of the surface modified particles (sample 7), the non-treated TiCN nanopowders as well as the REOSPERSE 205 surfactant used in the case of sample 7

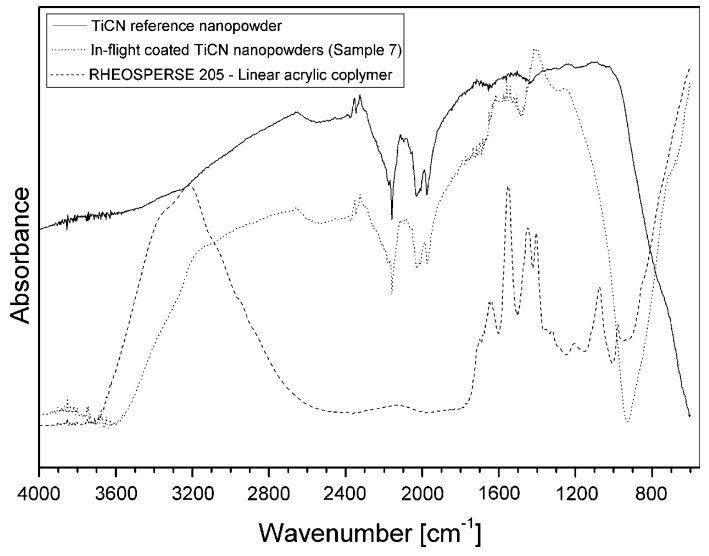




\section{Ex-Situ Coated TiCN Nanopowders}

A comparative study has then been performed on raw TiCN nanopowders produced with the inductively coupled plasma process coated ex-situ with the same linear acrylic copolymer as used for sample 7. Therefore, REOSPERSE 205 solution with different concentrations $(0.1,0.5$ and $1 \mathrm{wt} \%)$ was added at room temperature to the ICP produced TiCN nanoparticles and subsequently treated by ultrasonication as previously described. The results of the PCS measurements are presented in Fig. 11 together with the raw TiCN nanopowders as well as the in situ in-flight coated sample 7.

The ex-situ addition of the surfactant allows measuring particles with an average size around $50 \mathrm{~nm}$ after only $5 \mathrm{~min}$ low energy sonication, whereas for the in situ functionalised particles of sample 7 agglomerates with an average size above $200 \mathrm{~nm}$ were detected. No difference is observed between 0.1 and $0.5 \mathrm{wt} \%$ of surfactant after dispersion in the ultrasonic bath for $5 \mathrm{~min}$. However using the high energy ultrasonic probe for $2 \mathrm{~min}$ seems not sufficient to break down the agglomerates of the particles coated with a low surfactant concentration. Indeed the TiCN powders mixed with $0.1 \mathrm{wt} \%$ of surfactant still build agglomerates with sizes above $100 \mathrm{~nm}$. On the contrary the nanopowders treated with $0.5 \mathrm{wt} \%$ of surfactant de-agglomerate further and primary particles with a size around $20 \mathrm{~nm}$ were measured. The highest surfactant concentration $(1 \mathrm{wt} \%)$ investigated, leads to a mean particle size around $30 \mathrm{~nm}$ under the same sonication conditions. This seems to confirm that an optimal surfactant concentration is required for a better dispersability of the nanopowders.

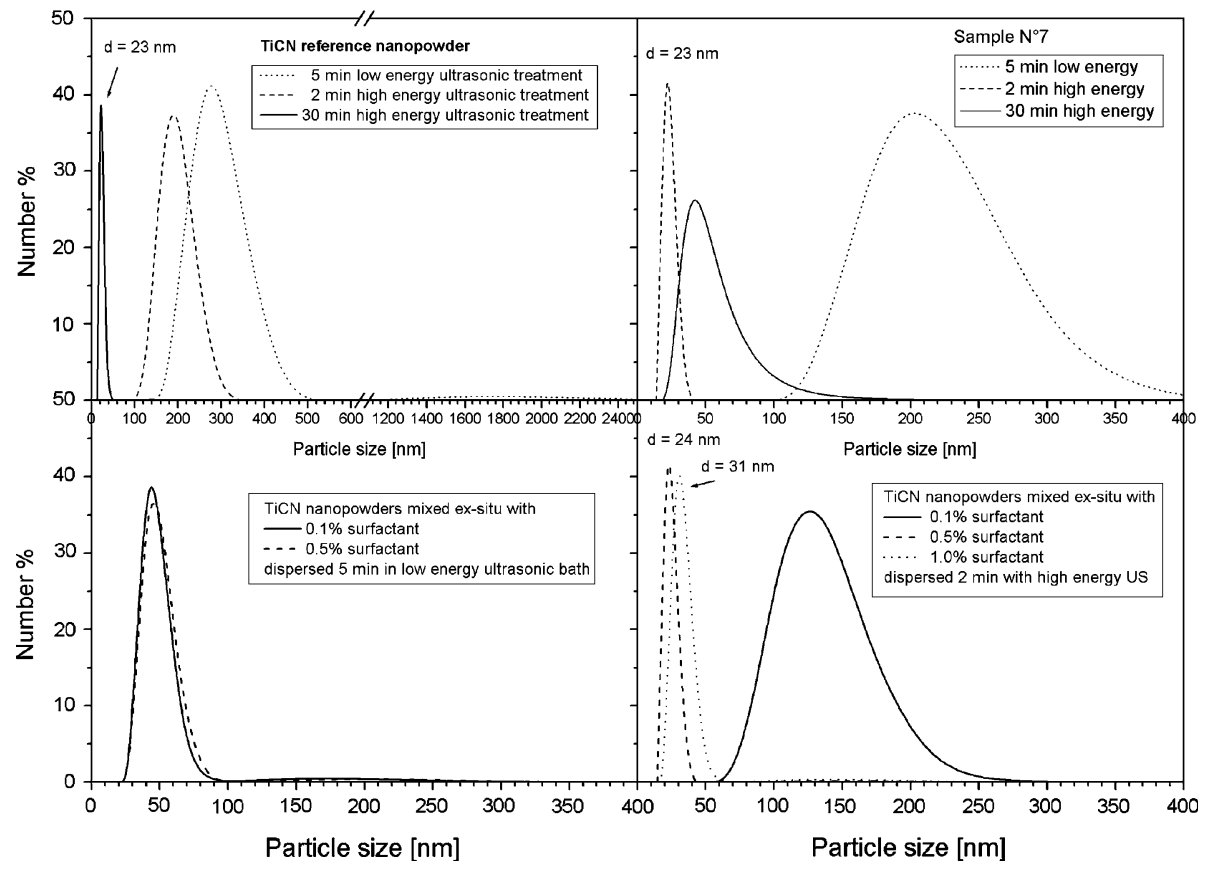

Fig. 11 Particle size distributions of the TiCN nanopowders after their synthesis without any addition of surfactant (top left), after in situ in-flight coating (top right) and ex-situ coating (bottom) as a function of the sonication route and duration 
Fig. 12 FTIR absorbance of the TiCN nanopowders coated ex-situ with $0.5 \mathrm{wt} \%$ of the REOSPERSE 205 solution

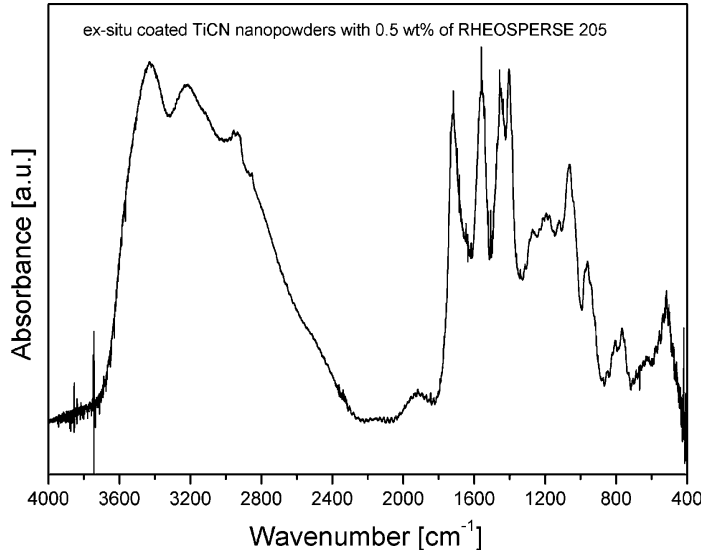

The FTIR spectra of the ex-situ surface modified TiCN nanopowders with $0.5 \mathrm{wt} \%$ of surfactant is presented in Fig. 12.

When compared to FTIR spectra in Fig. 10, this spectrum (Fig. 12) shows that the concentration of the surfactant deposited in situ in-flight on the nanoparticle surface is lower than $0.5 \mathrm{wt} \%$. The characteristic $\mathrm{C} \equiv \mathrm{N}$ bands observed around $2,000 \mathrm{~cm}^{-1}$ for the TiCN raw nanoparticles are indeed very weak compared to the surfactant bands. The ex-situ coating, as well as the ultrasonic treatment, does not seem to induce any modification of the IR signature of the linear acrylic copolymer, while the surfactant appears to be modified when deposited in situ in-flight.

\section{Discussion and Conclusion}

TiCN nanopowders with a particle size around $20 \mathrm{~nm}$ and a particle size distribution from 10 to $50 \mathrm{~nm}$ could be produced in an inductively coupled thermal plasma reactor. Effectively dispersed primary nanoparticles could be indeed characterized by SEM as well as by PCS after high energy sonication. The plasma processing conditions prevent then the formation of hard agglomerates. It has been already demonstrated that the adaptation of the quenching position and gas flow rate has an influence on the particle size [9, 12, 15-18]. In this study, the quenching conditions used should additionally decrease the surface temperature of the nanoparticles below their sintering temperature as no sintering necks are formed; the PCS measurements are in agreement with the microscopic observations of the primary particles. Beside the quenching conditions, the large volume of the reactor chamber promotes a dilution of the nanoparticles and then decreases their collision probability. Agglomerates are formed if the coalescence or sintering time is larger than the collision time [19]. By reducing the collision probability, the agglomeration formation is hampered. This has been proven with the in-line sampling experiments where dispersed nanoparticles were deposited on a TEM grid inserted in the reactor below the quenching device at different positions. The produced nanoparticles are then dispersed in the gaseous phase until a certain distance, exactly before the particle loaded gas flow is restricted in the cone shape of the reactor before the collection unit. It is obvious that the nanoparticles agglomerate as they reach the filter surface and consequently the collected nanopowders form generally, at least, soft agglomerates. The geometry of the used reactor and the 
presence of view ports at different heights allow then the introduction of other precursors while the particles are dispersed in the chamber, before the agglomeration takes place.

The possibility to coat in-flight the surface of primary nanoparticles by a polymer has been demonstrated. The surfactant solution was introduced in the synthesis reactor in the form of droplets using a carrier gas and a single injector at two different positions where the nanoparticles were still dispersed in the gas phase at a moderate temperature to avoid thermal degradation of the surfactant molecules.

Even if this study was a first screening of different types of surfactant able to deposit on the surface of TiCN nanoparticles, some first conclusions and trends could be drawn.

First, the injection of the surfactant solution does not disturb the plasma synthesis, the same average particle sizes could be observed for the surface modified and the raw nanoparticles. The formed agglomerates are mainly soft, maintained only by van der Waals forces. Second, some polymer molecules have been identified at the surface of the TiCN nanoparticles by infrared spectroscopy after in situ in-flight and also ex-situ coating. At the surfactant solution injection points, the gas temperature in the reaction chamber is already above $100^{\circ} \mathrm{C}$ and rise up to about $300^{\circ} \mathrm{C}$ in the centre of the chamber. Moreover the nanoparticles have also a temperature above $200-300^{\circ} \mathrm{C}$ when they reach the coating zone. It is then expected that most of the droplets evaporate completely in the gas phase or, at least, at the nanoparticle surface. The surfactant layer would then form by condensation of this vapour. The ex-situ coating is performed by adsorption of the organic molecules at the surface of the nanoparticles. For the particles coated in the plasma processing chamber, the IR signature of the polymer detected is slightly different than the one from the initial copolymer. An explication would be that surfactant molecules undergo a thermal degradation either during the injection in the hot plasma chamber or due to a thermal reaction at the surface of the nanoparticles. On the contrary, the IR bands observed on the ex-situ coated nanoparticles are similar to those measured from the surfactant solutions. No reaction takes then place between the copolymer and the TiCN nanoparticles at least at room temperature.

Depending on the coating conditions more or less high ultrasonic energy had to be used to break down the soft agglomerates into primary particles. This shows the importance of the sample preparation for the size distribution measurements of primary particle by photon correlation spectroscopy and the difficulty to define a measuring procedure for nanoparticles. In the present study, we could demonstrate that low energy sonication never led to the detection of primary particles, even if a better dispersion was achieved for ex-situ surfactant coated nanopowders. Nevertheless, most of the powders could be dispersed with a high energy sonication for $30 \mathrm{~min}$ treatment. Following the evolution of the agglomerate size distribution with the sonication time, it appears that a compromise has to be found concerning the concentration of the surfactant at the surface of the nanoparticles; excess of copolymer tends to hinder the further dispersion of the primary particles whereas too low coverage of the nanoparticles with the surfactant requires more ultrasonic energy to disperse them in water. This observation is confirmed also by the ex-situ coating experiments where the easiest nanoparticles dispersability was detected for the intermediate surfactant concentration of $0.5 \mathrm{wt} \%$. For large surfactant solution concentrations, the microscale droplets should intercept many nanoparticles. Moreover, the evaporation should be less efficient; the gas phase should be further cooled. A liquid phase may then condense directly onto many particles leading to agglomeration and coverage of the agglomerates with the polymer. These covered agglomerates are then more difficult to disperse. No observation could be done however concerning a thickness of the polymer layer depending on the relative quantity of surfactant solution introduced in the reaction chamber. 
Similar dispersability, i.e. primary particles around $20 \mathrm{~nm}$ observed already after $2 \mathrm{~min}$ of high energy US probe treatment, could be found also for one sample coated in situ in-flight (sample 7). This sample was coated with a linear acrylic copolymer injected at the highest investigated position and it has a relatively moderate surfactant concentration. In this case, the thermal degradation of the surfactant does not seem to affect the particle dispersability. A longer duration of the sonication led however to a re-agglomeration of the nanoparticles.

In spite of the thermal conditions encountered by the species in inductively coupled plasma gas phase synthesis process, it was found possible to deposit an organic surfactant layer on the surface of the TiCN nanoparticles before they start to agglomerate in the gas flow and/or on the filters. This in situ in-flight coating of the TiCN nanopowders was found almost as efficient as an ex-situ addition of the same surfactant. Therefore, the possibility to obtain dispersible nanoparticles directly after their production with no need for subsequent addition of dispersing agent allows reducing the number of the process steps. The integration of many powder processing steps is important considering economical aspects and also safety issues as each manipulation of nanopowders presents potentially a risk of nanoparticles emission in the environment. This technique could be of high interest for many applications requiring large scale nanopowders such as in the ceramic industry or in any context where individualized nanoparticles suspensions are required.

Acknowledgments The authors thank Coatex SAS (France) for the supply of the surfactant solutions. A. Habert and A. Casanova (LFP) are also acknowledged for the suspensions preparation and the PCS, FTIR and TGA measurements.

\section{References}

1. Siegel RW (1993) Mater Sci Eng A 168:189

2. Kruis FE, Fissan H, Peled A (1998) J Aerosol Sci 29:511

3. German RM (2005) Powder metallurgy and particulate materials processing. Metal Powder Industries Federation, Princeton

4. Esawi AMK, El Borady MA (2008) Compos Sci Technol 68:486

5. Piao L, Lee KH, Kwon WJ, Kim SH, Yoon S (2009) J Colloid Interface Sci 334:208

6. Siddiqui SW, Unwin PJ, Xu Z, Kresta SM (2009) Colloids Surf A Physicochem Eng Asp 350:38

7. Chinthamanipeta PS, Kobukata S, Nakata H, Shipp DA (2008) Polymer 49:5636

8. Conley RF (1996) Practical dispersion. VCH Publishers Inc., New York

9. Wang Y, Zhang J, Shen X, Shi C, Wu J, Sun L (2006) Mater Chem Phys 98:217

10. Qin C, Coulombe S (2006) Mater Lett 60:1973

11. Hakim LF, Blackson JH, Weimer AW (2007) Chem Eng Sci 62:6199

12. Leparoux M, Schreuders C, Fauchais P (2008) Adv Eng Mater 10(12):1147

13. Leparoux M, Loher M, Schreuders C, Siegmann S (2008) Powder Technol 185:109

14. Leparoux M, Paris S, Kihn Y, Schreuders C (2008) Int J Refract Metals Hard Mater 26:277

15. Leconte Y, Leparoux M, Portier X, Herlin-Boime N, Reynaud C (2008) Plasma Chem Plasma Proc 28:233

16. Ye R, Li J-G, Ishigaki T (2007) Thin Solid Films 515:4251

17. Goortani BM, Prouls P, Xue S, Mendoza-Gonzales NY (2007) Powder Technol 175:22

18. Shigeta M, Watanabe T, Nishiyama H (2004) Thin Solid Films 457:192

19. Friedlander SK (2000) In: Smoke, Dust, and Haze, 2nd edn. Oxford University Press 